\title{
The Impact of Variable Inlet Mixture Stratification on Flame Topology and Emissions Performance of a Premixer/Swirl Burner Configuration
}

\author{
P. Koutmos, G. Paterakis, E. Dogkas, and Ch. Karagiannaki \\ Laboratory of Applied Thermodynamics, Department of Mechanical and Aeronautical Engineering, University of Patras, \\ 26500 Patras, Greece \\ Correspondence should be addressed to P. Koutmos, koutmos@mech.upatras.gr
}

Received 4 July 2012; Accepted 30 August 2012

Academic Editor: Constantine D. Rakopoulos

Copyright ( $) 2012$ P. Koutmos et al. This is an open access article distributed under the Creative Commons Attribution License, which permits unrestricted use, distribution, and reproduction in any medium, provided the original work is properly cited.

The work presents the assessment of a low emissions premixer/swirl burner configuration utilizing lean stratified fuel preparation. An axisymmetric, single- or double-cavity premixer, formed along one, two, or three concentric disks promotes propane-air premixing and supplies the combustion zone at the afterbody disk recirculation with a radial equivalence ratio gradient. The burner assemblies are operated with a swirl co-flow to study the interaction of the recirculating stratified flame with the surrounding swirl. A number of lean and ultra-lean flames operated either with a plane disk stabilizer or with one or two premixing cavity arrangements were evaluated over a range of inlet mixture conditions. The influence of the variation of the imposed swirl was studied for constant fuel injections. Measurements of turbulent velocities, temperatures, $\mathrm{OH}^{*}$ chemiluminescence and gas analysis provided information on the performance of each burner set up. Comparisons with Large Eddy Simulations, performed with an 11-step global chemistry, illustrated the flame front interaction with the vortex formation region under the influence of the variable inlet mixture stratifications. The combined effort contributed to the identification of optimum configurations in terms of fuel consumption and pollutants emissions and to the delineation of important controlling parameters and limiting fuel-air mixing conditions.

\section{Introduction}

Flow recirculation is exploited within combustion systems to promote fuel-air mixing, flame stability, and high efficiency over a wide range of operating conditions for modern power systems [1-3]. Plane or axisymmetric bluff-body stabilizers are popular arrangements for experimental and computational flame stabilization studies under nonpremixed $[2,4]$ and fully premixed configurations $[5,6]$. Swirl motion is an equally common method of stabilization in industrial burners. Plane swirl can be regulated to create a free standing central recirculation zone (CRZ) for aerodynamic flame holding, while in combination with a bluff body allows for improved stability and emissions within reasonable residence times $[2,7]$.

In recent years, driven by more stringent regulations, new combustion technologies based on partially premixing the reactants to establish stratified, lean or ultra-lean operating conditions, have emerged as promising methodologies in the effort to achieve target efficiencies and emissions [3]. Nonuniformities in the fuel-air ratio offer design flexibility and are often exploited for a more effective management of fuel lean operation through mixture stratification [8]. However, these are also expected to lead to a spatially varying combustion performance particularly in relation to the local large and small scale equivalence ratio variations and the interaction of the developing flame front with the local turbulence within the context of a narrower stability margin $[9,10]$. All these are significant aspects affecting early design procedures and therefore, deserve further investigation in a variety of possible fuel-air placement settings and stabilizing geometries [11-13].

Within the above context the present work investigates the characteristics of stratified premixed flames, established 
by upstream fuel injection and staged premixing within assemblies employing one, two, or three, successively placed, concentric disks and stabilized in the afterbody disk vortex region. The adopted set up bears some similarity to the trapped vortex combustor concept [14]. This geometry has been found attractive for fuel injection, ignition, and flame holding internal to the cavities, although it can comprise an equally convenient set up for cold fuel-air staging and premixing. Here, no reaction occurs within the cavities; these are merely exploited as a mixture preparation device supplying the primary recirculation with a radially stratified equivalence ratio [11-13] that is being regulated by the upstream in-cavity fuel injection. In the present work, arrangements with one, two, or three disks resulting in none, one, or two cavity assemblies are also operated with a surrounding swirl co-flow to study the interaction between the primary recirculaing afterbody flame and the swirling field.

The complete three-disk double-cavity arrangement has been previously studied by Xiouris and Koutmos [11, 15]. Here, the relative merits and the suitability of adopting none, one, or two premixer cavities upstream of the stabilizing afterbody is parametrically investigated for various swirl intensities and fuel-air settings. Such information is useful and may be required when there are constraints in the use of the full double-cavity system or when existing systems are upgraded, modernized, or retrofitted (e.g., [9]). Turbulent velocities, temperatures, flame structure images, and pollutant emissions were obtained using laser velocimetry, thin digitally compensated thermocouples, chemiluminescence imaging, and exhaust gas analysis to quantify the relative burner performance. Accompanying large eddy simulations were undertaken using either the eddy dissipation concept [16] or a quasilaminar turbulent chemistry description (e.g., [6]) together with an 11-step global mechanism for propane oxidation and $\mathrm{NO}_{x}[17,18]$. The parametric comparisons against experimental data helped to delineate important controlling parameters and fuel-air conditions that influence the performance of each premixer/burner configuration. Initial successful cold flow simulations allowed for a meaningful extension of the basic modelling methodology to the reacting wakes.

\section{Experimental Methodology}

2.1. Premixer/Burner Configurations. The combustion tunnel facility and the premixer/burner configuration set up at the Laboratory of Applied Thermodynamics are shown in Figures 1(a), 1(b), and 1(c) [11]. The premixer/burner assemblies (Figure 1(c)) were composed of one, two, or three-disk-shaped axisymmetric bluff bodies $\left(D_{b}=25 \mathrm{~mm}\right)$, connected along their axis with a central shaft made up of a hollow fuel supplying tube $\left(D_{p}=10 \mathrm{~mm}\right)$. Arrangements with two cavities (employing all three disks, i.e., A (afterbody), B (fuel injector), and C (forebody) defined as set up no. 3), one cavity (employing disks A and B only_set up no. 2), or without cavity (using only disk A as injector-set up no. 1) were parametrically investigated. A sketch of the simulated flow patterns within the double cavity system for a lean flame without swirl together with the possible fuel injection placement positions is depicted in Figure 2. The central tube $\left(D_{c}=0.052 \mathrm{~m}\right)$ can supply either a mixture of propane and air or plane air. In either cases, the primary stabilization region is fuelled with a radially stratified equivalence ratio gradient that can be regulated both via the central propane-air mixture supply and the fuel injection levels from the injecting disks (Figure 2).

For the present study, the central tube supplies plane air, and the fuel is injected either through disk A (for the single-disk set up no. 1) or through disk B (set ups no. 2 and 3 ) and is then mixed with the incoming central tube air. Specifically, propane is supplied through the internal hollow of the injecting disk (A or B depending on set up) and then injected through an annular $1 \mathrm{~mm}$ slot (with an $8 \mathrm{~mm}$ radial offset from the axis), against the central air (for the singledisk and the single-cavity set ups) or within the primary cavity (double-cavity set up).

This maintains an afterbody equivalence ratio $(\Phi)$ gradient between a $\Phi_{\min } \approx 0.1$ to 0.4 and a $\Phi_{\max } \approx 0.5$ to 1.85 (Figure 2) as measured with flame ionization detection from local gas samples at the afterbody exit; the exact levels depend on the burner set up and the injected fuel adjustment. A typical range of such values for a lean fuel setting is shown in Figure 3; this inlet stratification will be discussed further in Section 4. In the single- and double-cavity cases a stratified mixture profile can be maintained across the leading edge of the flame front stabilization position close to the afterbody burner rim. A significantly lower level of premixing is achieved by the single disk, which operates close to an almost non premixed mode with a much greater spread of $\Phi$ values. The global $\Phi$, based on the total mass flows of fuel and central air together with the range of investigated conditions is shown in Table 1 and is maintained for all cases at 0.18 . The blockage ratio (BR) of the central annular jet was BR $=\left(D_{b} / D_{c}\right)^{2}=0.23$. The Reynolds number based on the afterbody diameter and the central air velocity $\left(U_{c}\right)$ was maintained at 8000 .

The cavity lengths were chosen from past experience and in line with aerodynamic design values established in previous studies [14] but also according to performed isothermal mixing [11] and reacting computations [15]. The length of the secondary cavity was also dictated by the requirement for operation free from flame flashback. This is interrelated to the balance between the mixing time and the autoignition time of the evolving mixture as it approaches the leading edge of the flame front stabilized at the afterbody rim.

The burner was operated with a surrounding swirling coflow supplied via a concentric tube of $D_{s}=85 \mathrm{~mm}$ diameter (Figures 1(a) and 1(b)) and introduced aerodynamically (e.g., [2]) $300 \mathrm{~mm}$ upstream of the afterbody exit, via four circumferential tangential ports inclined $15^{\circ}$ upwards (Figure 1(b)). An annulus air co-flow $\left(D_{e}=141 \mathrm{~mm}\right)$ shielded the system operation from external disturbances (Figure 1(a)).

2.2. Experimental Methods. Each burner set up was initially investigated under isothermal operation to establish the 


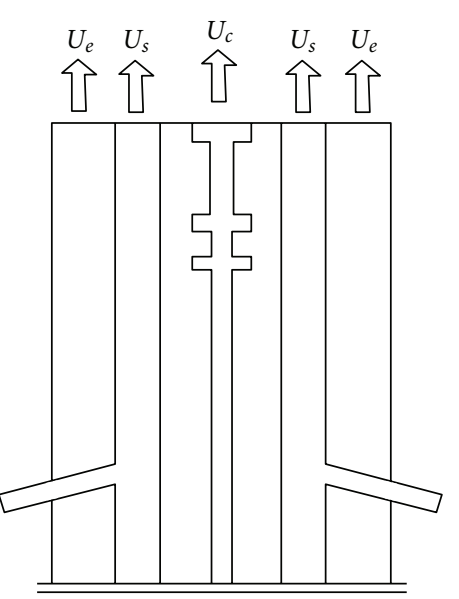

(a)

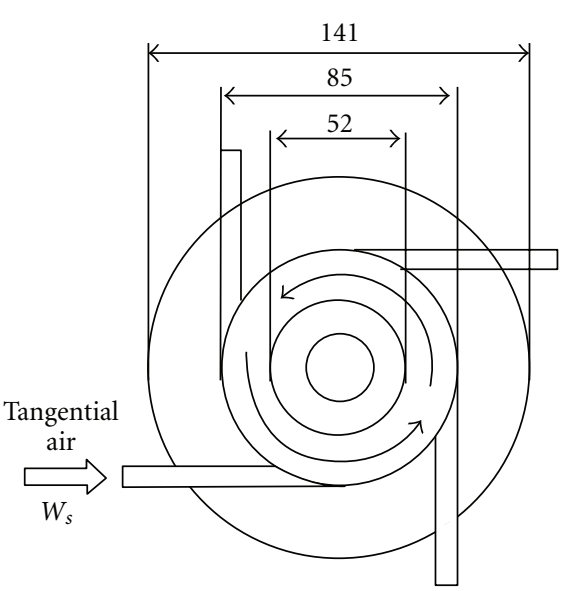

(b)

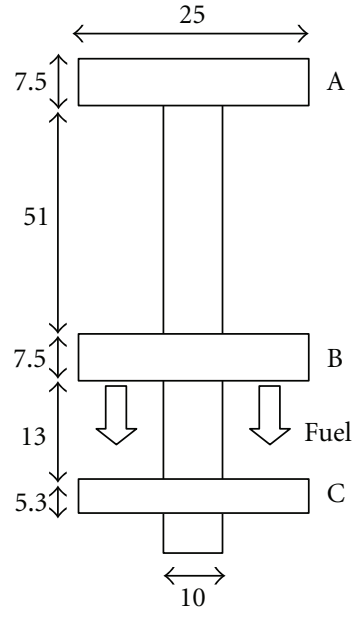

(c)

FIgURE 1: Combustion tunnel and premixer/burner configurations.
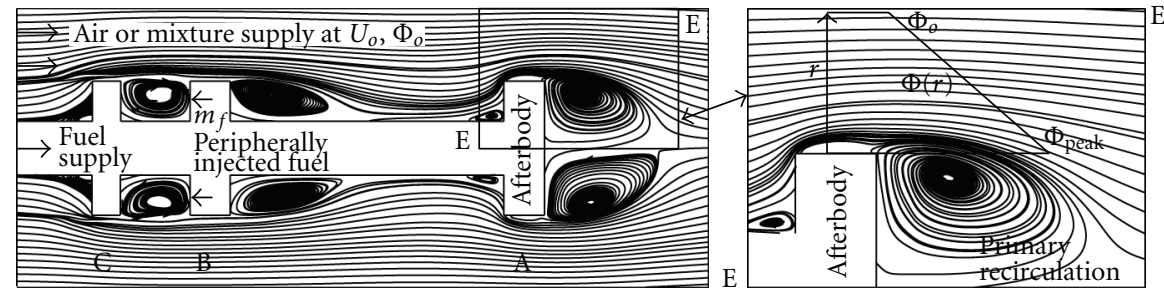

FIGURE 2: Fuel, air flows, and mixture placement at flame stabilizer inlet.

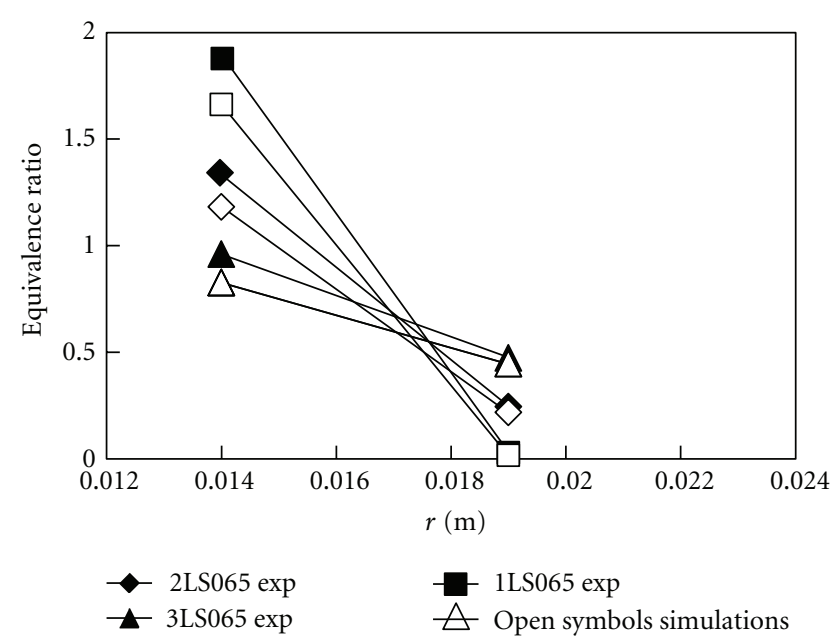

FIgURE 3: Equivalence ratio gradients at the exit from the different premixing assemblies.

mixing topologies that sustain the reacting fields presented below. All flames studied were lying within the stable operation envelope of the burner, as shown in the burner stability diagram (Figure 4). Flame nomenclature is denoted on the basis of the cavity system, the swirl strength, and the level of the operating equivalence ratio. For instance,

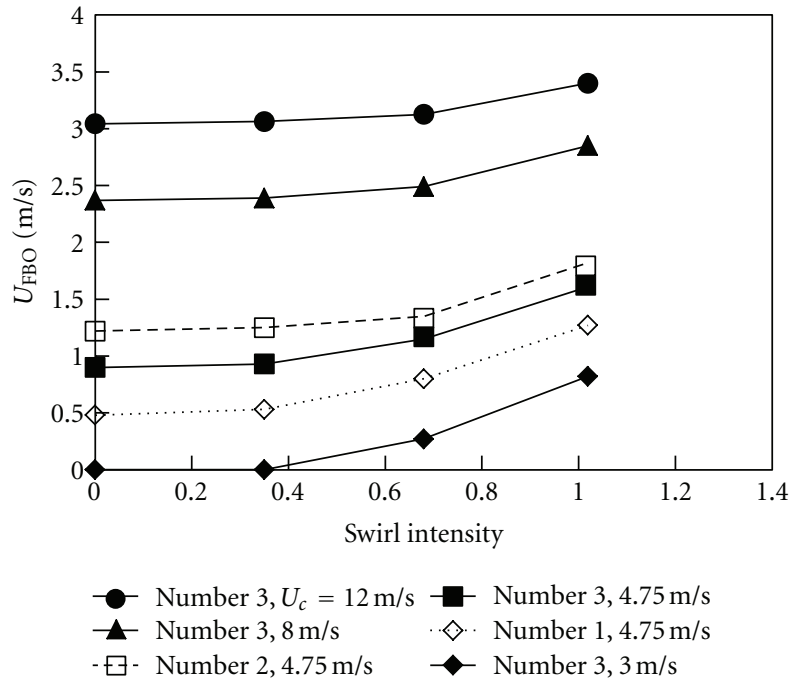

FIGURE 4: Stability performance of the three premixer/burner set ups at various operating conditions.

3LS065 denotes a flame established by a (3) disk, that is, double-cavity arrangement (set up no. 3), with a (L)ean $\Phi$ and a (S)wirl strength $S=0.65$; 1LS065 denotes the above flame for the single-disk arrangement. Here, lean flames are studied for each burner set up operated at swirl intensities 
TABLE 1: Investigated premixer/burner conditions.

\begin{tabular}{lcccccccc}
\hline Case & $U_{\text {Fuel }}$ & $U_{\text {FBO }}$ & $\delta(\%)$ & Swirl & $T_{\max }(\mathrm{K})$ & $L_{f} / D_{b}$ & $L_{R} / D_{b}$ & $\mathrm{Ka}$ \\
\hline IIS000 & 0 & 0 & 0 & 0 & 300 & 0 & 1.35 & 0 \\
1IS065 & 0 & 0 & 0 & 0.65 & 300 & 0 & 1.236 & 0 \\
2IS000 & 0 & 0 & 0 & 0 & 300 & 0 & 1.27 & 0 \\
2IS065 & 0 & 0 & 0 & 0.65 & 300 & 0 & 1.15 & 0 \\
3IS000 & 0 & 0 & 0 & 0 & 300 & 0 & 1.175 & 0 \\
3IS065 & 0 & 0 & 0 & 0.65 & 300 & 0 & 1.125 & 0 \\
1LS000 & 1.75 & 0.50 & 249 & 0 & 2181 & 6.4 & 1.98 & 12 \\
1LS065 & 1.75 & 0.80 & 118 & 0.65 & 2122 & 5 & 1.78 & 28 \\
2LS000 & 1.75 & 1.22 & 44 & 0 & 1995 & 5.35 & 1.57 & 10 \\
2LS065 & 1.75 & 1.35 & 30 & 0.65 & 1975 & 3.85 & 1.37 & 26 \\
3LS000 & 1.75 & 0.90 & 94 & 0 & 1981 & 5.10 & 1.58 & 10 \\
3LS065 & 1.75 & 1.15 & 52 & 0.65 & 1925 & 3.75 & 1.45 & 25 \\
\hline
\end{tabular}

(1) $\delta:\left(m_{\text {Fuel }}-m_{\mathrm{FBO}}\right) / m_{\mathrm{FBO}}(\%)$, (FBO: fuel flow at blow off).

(2) $T_{\max }$ : maximum measured wake temperature.

(3) $L_{f}$ : visible flame length, $L_{R}$ : measured primary recirculation length.

(4) Central air velocity, $U_{c}=4.75 \mathrm{~m} / \mathrm{s}$, co-flow velocity, $U_{s}=10.5 \mathrm{~m} / \mathrm{s}$, and $\operatorname{Re}_{D_{b}}: 7985$ for all cases.

(5) $\Phi_{\text {Global }}: 0.18$, global equivalence ratio, based on mass flow rates of fuel and central air supplies.

(6) MR: 0.14, momentum ratio between central fuel-air stream and swirl co-flow.

(7) Power, $P: 6.73 \mathrm{~kW}$ for all flames.

of 0 and 0.65 (Table 1), while the impact of increasing the swirl or decreasing the fuel level is also assessed for optimum configurations. The proximity to lean blow off (LBO) for each studied flame is given by the parameter, $\delta$, defined as, $\delta=\left(m_{\text {Fuel }}-m_{\text {Fuel, LBO }}\right) / m_{\text {Fuel, LBO }}$, with $m_{\text {Fuel, LBO }}$ being the limiting mass flow of the fuel. The reported swirl numbers $(S$, Table 1) were determined as the ratio of the mean tangential $\left(W_{S}\right)$ to the mean axial $\left(U_{S}\right)$ component in the swirl stream, both evaluated by integration of the corresponding laser Doppler velocimetry (LDV) profiles at the exit plane of the surrounding swirl tube.

Turbulent velocities, temperatures, flame structure images, and major pollutant concentrations were obtained using laser velocimetry, thin digitally compensated thermocouples, chemiluminescence imaging, and gas analysis. Profiles of the time-mean axial and tangential velocities and statistics were obtained with a single-component 2 Watt Argon-Ion laser and fiber optics linked to TSI transmitting and receiving optics. $\mathrm{MgO}$ particles seeded uniformly the fuel and air streams to minimize bias errors. The filtered Doppler signals were processed by a TSI frequency counter (1980B). Mean and statistical values were postprocessed from 20480 data weighted by the time between particles to correct for velocity bias.

Temperatures were measured with Pt-Pt/10\%Rhuncoated beaded thermocouples of 50 to $75-\mu \mathrm{m}$-diameter wire. The thermocouple output was interfaced to a DaqTemp 7A Omega card while its time constant was estimated using an FFT algorithm to obtain the frequency spectrum, correct the amplitude and phase of the signal, and reconstruct part of its spectrum (up to about 50-60\%) to calculate the fluctuating values $[11,19]$. No radiation correction was applied but for propane flames and uncoated wires, the systematic error in the mean can be up to $10 \%$ at $1900 \mathrm{~K}$ $[11,17]$. Mean and rms temperatures were derived at a sampling frequency of $400 \mathrm{~Hz}$. Maximum uncertainties in the velocities were less than $8.5 \%$ in the mean and less than $15 \%$ in the rms. Uncertainties in the estimation of the thermocouple time constant are rather unimportant for the mean temperature, but according to the present compensation procedure it may affect the variance by between $20-30 \%$ [11, 19].

A quartz microprobe was used to obtain samples at the afterbody annular exit, and flame ionization detection (FID) was employed to measure the concentration of the hydrocarbon and allow the evaluation of the equivalence ratio levels. The burner global emissions were evaluated with exhaust gas analysis. An exhaust probe, placed downstream of the burner exit, extracted flue gas and measured species $\left(\mathrm{NO}_{x}, \mathrm{CO}, \mathrm{CO}_{2}\right.$, and $\left.\mathrm{O}_{2}\right)$ concentrations with a Kane-May KM9106 Quintox analyzer. The sample gases drawn from the combustion zone through a stainless-steel probe, were cooled, dried, and then transferred to the analyzer. Gas samples were obtained in nine radial positions spanning a distance from the axis to the swirler radius, and this rake was repeated at four circumferential positions $90^{\circ}$ apart $[11,17]$. The mean of these values is reported in Section 4.

Flame, $\mathrm{OH}^{*}$, and $\mathrm{CH}^{*}$ chemiluminescence (CL) images were obtained using a LaVision FlameMaster imaging system consisting of a CCD camera (E-lite 2M), an image intensifier IRO unit, a camera optical filter at $307 \pm 10 \mathrm{~nm}$, and an achromat lens for $\mathrm{OH}^{*}$ imaging. $\mathrm{OH}^{*}$ and $\mathrm{CH}^{*} \mathrm{CL}$ measurements are considered good markers of the flame front since, for example, the electronically excited $\mathrm{CH}^{*}$ radical exists only in the flame front. Image acquisition (lineof-sight technique) and data reduction were performed using the DaVis $8.0 \mathrm{~m}$ software from LaVision. The background image, taken under the same integration time and gain of the measured images, was subtracted from the originals. Averages were produced from 300 instantaneous images recorded at 16 to 58 frames per second depending on the binning and the interrogation window [17]. The signal-tonoise ratio of the instantaneous images was better than $8: 1$, and the exposure time was $2.1 \mathrm{~ms}$. The maximum CCD chip resolution was $1626 \times 1236$ pixels. Intensifier gate times of $100 \mu$ s were used for the measurements with a gain of $65 \%$.

\section{Simulation Models}

3.1. Aerodynamic Model. Large Eddy Simulations (LES) of the flows were performed using the commercial code Fluent (Ansys Inc.). It offered mesh adaption flexibility near the slotted fuel injector and facilitated the exploitation of reduced chemistry within the context of an adequate modelling scheme for the turbulent reactions. Within the LES, the flow variables, $F$, can be decomposed into resolvable 
TABLe 2: $\mathrm{C}_{3} \mathrm{H}_{8} / \mathrm{NO}_{x}$ global mechanism.

\begin{tabular}{|c|c|c|c|c|}
\hline Rxn. no. & Reaction & A & $E_{a}$ & $n$ \\
\hline 1 & $\mathrm{C}_{3} \mathrm{H}_{8}{ }^{0.6}+\mathrm{O}_{2}+\mathrm{H}_{2} \mathrm{O}+2 \mathrm{H} \rightarrow 3 \mathrm{CO}+6 \mathrm{H}_{2}$ & $5 e+15$ & $4.06 e+07$ & 0 \\
\hline $2 \mathrm{f}$ & $\mathrm{CO}^{0.8}+\mathrm{H}_{2} \mathrm{O}^{1.25} \rightarrow \mathrm{CO}_{2}+\mathrm{H}_{2}$ & 33000 & -3100000 & 1.65 \\
\hline $2 b$ & $\mathrm{CO}_{2}{ }^{0.8}+\mathrm{H}_{2}{ }^{1.15} \rightarrow \mathrm{CO}+\mathrm{H}_{2} \mathrm{O}$ & 4100000 & -8971000 & 1.14 \\
\hline $3 \mathrm{f}$ & $3 \mathrm{H}_{2}{ }^{1.15}+\mathrm{O}_{2}{ }^{0.65} \rightarrow 2 \mathrm{H}_{2} \mathrm{O}+2 \mathrm{H}$ & $1.08 e+12$ & $8 e+07$ & -0.7 \\
\hline $3 b$ & $2 \mathrm{H}_{2} \mathrm{O}^{1.25}+2 \mathrm{H}^{0.75} \rightarrow 3 \mathrm{H}_{2}+\mathrm{O}_{2}$ & $1.5 e+10$ & $1.0476 e+08$ & -0.1 \\
\hline 4 & $2 \mathrm{H}+\mathrm{M}_{4} \rightarrow \mathrm{H}_{2}$ & $3.8 e+09$ & 0 & -1.2 \\
\hline 5 & $\mathrm{C}_{3} \mathrm{H}_{8}+\mathrm{H}_{2} \mathrm{O}+2 \mathrm{H} \rightarrow \mathrm{C}_{2} \mathrm{H}_{2}+\mathrm{CO}+5 \mathrm{H}_{2}$ & $1.6 e+12$ & $1.26 e+07$ & 0 \\
\hline 6 & $\mathrm{C}_{2} \mathrm{H}_{2}+\mathrm{O}_{2} \rightarrow 2 \mathrm{CO}+\mathrm{H}_{2}$ & $1 e+11$ & $6 e+07$ & 0 \\
\hline 7 & $\mathrm{~N}_{2}+2 \mathrm{O}^{0.9} \rightarrow 2 \mathrm{NO}$ & $6 e+010$ & $3.14 e+08$ & 0.3 \\
\hline 8 & $\mathrm{NO}^{0.72}+\mathrm{O} \rightarrow 0.5 \mathrm{~N}_{2}+\mathrm{O}_{2}$ & $8 e+06$ & $1.65 e+08$ & 1 \\
\hline 9 & $2 \mathrm{O}+\mathrm{M}_{9} \leftrightarrow \mathrm{O}_{2}$ & $4 e+12$ & 0 & -1.25 \\
\hline $10 \mathrm{f}$ & $\mathrm{O}_{2}^{0.47}+0 \mathrm{H}_{2} \rightarrow 2 \mathrm{O}+0 \mathrm{H}_{2} \mathrm{O}$ & $4.5 e+12$ & $7.15 e+07$ & -0.7 \\
\hline $10 \mathrm{~b}$ & $2 \mathrm{O}+0 \mathrm{H}_{2} \mathrm{O} \rightarrow \mathrm{O}_{2}+0 \mathrm{H}_{2}$ & $1.9 e+09$ & -748260 & -0.32 \\
\hline $11 \mathrm{f}$ & $0 \mathrm{C}_{3} \mathrm{H}_{8}{ }^{0.02}+\mathrm{N}_{2}{ }^{1.16}+\mathrm{O}_{2}^{0.5}+0 \mathrm{H}_{2} \mathrm{O}^{0.5} \rightarrow 2 \mathrm{NO}$ & $4 e+09$ & $2.3 e+08$ & 0.2 \\
\hline $11 \mathrm{~b}$ & $0 \mathrm{C}_{3} \mathrm{H}_{8}{ }^{0.05}+2 \mathrm{NO}^{1.1} \rightarrow \mathrm{N}_{2}+\mathrm{O}_{2}$ & $2.16 e+08$ & $6.7 e+07$ & 0 \\
\hline
\end{tabular}

Rate constants are in $A T^{\mathrm{b}} \exp \left(-E_{a} / R T\right)$ format. Units are $\mathrm{Kg}$ mol, $\mathrm{m}, \mathrm{s}, \mathrm{K}$, and J.

$\mathrm{M}_{4}=2.4 \mathrm{CO}_{2}+1.2 \mathrm{CO}+16 \mathrm{H}_{2} \mathrm{O}+2.5 \mathrm{H}_{2}$.

$\mathrm{M}_{9}=3 \mathrm{C}_{3} \mathrm{H}_{8}+0.35 \mathrm{O}_{2}+3.6 \mathrm{CO}_{2}+1.75 \mathrm{CO}+15 \mathrm{H}_{2} \mathrm{O}+2.4 \mathrm{H}_{2}+0.001 \mathrm{O}$.

$\widetilde{F}$ and subgrid $F^{\prime}$ scale quantities using a Favre-weighted filter, $\widetilde{F}=\overline{\rho F} / \bar{\rho}$. The equations describing the resolvable flow quantities are (e.g., $[6,15])$ as the following:

$$
\begin{gathered}
\frac{\partial \bar{\rho}}{\partial t}+\frac{\partial \bar{\rho} \tilde{u}}{\partial x_{i}}=0, \\
\frac{\partial \bar{\rho} \tilde{u}_{i}}{\partial t}+\frac{\partial\left(\bar{\rho} \tilde{u}_{i} \tilde{u}_{j}\right)}{\partial x_{j}} \\
=-\frac{\partial \bar{p}}{\partial x_{i}}+\frac{\partial}{\partial x_{j}} \tilde{\sigma}_{i j}+\frac{\partial}{\partial x_{j}} \tau_{i j}+\left(\bar{\rho}-\rho_{\infty}\right) g_{i},
\end{gathered}
$$

( $\rho, \mu, T$, and $u$ are the density, viscosity, temperature, and velocity of the gas, and $i=1,2$ in a Cartesian system $(x, y)) . \bar{p}=\bar{\rho} R_{u} \widetilde{T} \Sigma_{i} Y_{i} / M_{i}$, where $Y_{i}, M_{i}$, and $R_{u}$ are the mass fraction, the molecular weight of species $i$, and the universal gas constant. $\tilde{\sigma}_{i j}=\mu\left(\widetilde{S}_{i j}-(2 / 3) \widetilde{S}_{k k} \delta_{i j}\right)$, with $\left(\delta_{i j}=0\right.$ for $i \neq j, \delta_{i j}=1$ for $\left.i=j\right), \tau_{i j}$ are the SGS stresses, and $\tilde{S}_{i j}$ is the resolvable strain tensor. $\tau_{i j}$ is modeled as: $\tau_{i j}=-\bar{\rho} \overline{u_{i}^{\prime} u_{j}^{\prime}}=\mu_{t, i j}\left(\tilde{S}_{i j}-(2 / 3) \tilde{S}_{k k} \delta_{i j}\right)-(2 / 3) \bar{\rho} \tilde{k}_{s} \delta_{i j}$, where $k_{s}$ is the subgrid kinetic energy. The subgrid turbulent viscosity is calculated through the resolvable strain tensor: $\mu_{t, i j}=\widetilde{\rho}\left(C_{s} \Delta\right)^{2}\left(2 \widetilde{S}_{i j} \widetilde{S}_{i j}\right)^{1 / 2}$, where $\Delta$ is the filter length: $\Delta=$ $\sqrt{\Delta x_{i} \Delta y_{i} \Delta z_{i}}$.

Simulations employed a dynamic Smagorinsky constant, $C_{s}\left(0<C_{s}<0.23\right)$, and the SIMPLE algorithm for pressurevelocity coupling. Central differencing was used for all equations, while temporal integration was performed with a second-order procedure. The simple P-1 radiation model available in the software (Ansys Inc.) was adopted in the simulations.
3.2. Combustion Model. The adoption of a reduced chemistry scheme with a detailed transport model (kinetic theory) within a tractable turbulent combustion closure was considered beneficial in addressing the compositional stratification across the flame front anchoring region alongside the disk rim. The measurements suggested that the lower swirl flames $(S<0.55)$ lie in the thin reaction regime, while the higher swirl ultra-lean flames closer to extinction $(S>$ 0.8 ) are nearer the broken reaction zone boundary with Karlovitz numbers [3], $\left(\mathrm{Ka}=\tau_{\mathrm{ch}} / \tau_{k}\right.$, where $\tau_{\mathrm{ch}}$ and $\tau_{k}$ are the chemical and Kolmogorov timescales,) up to 80. The eddy dissipation concept (EDC, [16]) turbulent combustion model implemented in Fluent was deemed suitable to cover the range of conditions. Alternatively, the adoption of a quasilaminar reaction rate source term closure (e.g., [6]) produced similar results when used with the finest meshes.

Both turbulence/chemistry treatments were coupled to an eleven-step-reduced chemistry employing a 6-step global scheme for propane and a 5-step $\mathrm{NO}_{x}$ production scheme (using "tuned" thermal, $\mathrm{N}_{2} \mathrm{O}$, and prompt contributions) $[17,18]$. A detailed description of this mechanism is given in Table 2. The computation of the reaction rate source terms is accelerated with the ISAT algorithm [4] embodied in the solver.

3.3. Computational Details. Particular emphasis was given to the mesh adaption close to the small relative dimensions of the slot injector, (i.e., $0.001: 0.0125: 0.026) \leftrightarrow$ (peripheral injection slot: afterbody radius: central air supply tube radius), near the disk walls and along the flame front stabilizing shear layers emanating with the annular fuelair jet. Within the context of the commercial software and 
the available computational power hybrid, meshes were used. These were denser and partly unstructured near the slot injector, in the axis region and structured in all other locations with moderate expansion to the outlet. The meshes extended upstream to resolve the cavities, and the outlet was placed $16 D_{b}$ downstream of the afterbody.

Typical meshes of $1.35 \mathrm{M}$ cells were employed for the basic runs. No-slip boundary conditions were used near walls with the node close to the disk walls placed at $\Delta y / D_{b}=$ $0.00015\left(y^{+} \approx 0.45\right)$, while the law of the wall was applied elsewhere. Inlet conditions were taken from measurements, while a static pressure boundary condition was applied at the outlet and all outflowing quantities were extrapolated from the interior node. The LES quality was checked by calculating the resolved fraction of the turbulence energy, $k, R_{k}=$ $k_{\text {resolved }} /\left(k_{\text {resolved }}+k_{\text {subgrid scale }}\right)$ [20], which achieved values up to $95 \%$ within the primary reaction zone. Additionally, the influence of mesh resolution in the near wake region was tested by undertaking successive runs with different levels of grid refinement up to the available resources (with meshes of $0.48,0.79$ and 1.345 Mcells). These suggested that, between the medium and the finer grid, deviations in the first moment statistics were of the order of $5 \%$, while the disagreement in the higher-order statistics reached about 16\%. Run times on $362.83 \mathrm{GHz}$ processors (3 XEON 5660) and $243.33 \mathrm{GHz}$ processors (4 i7) run in parallel were about $25 \mathrm{sec}$ per inner iteration (including the chemistry integration time) for the finer mesh.

\section{Results and Discussion}

The flow topology established in the single-disk and the single- or the double-cavity set up is of importance for preconditioning the premixing effectiveness and the quality of the annular mixture gradient supplied into the primary reaction zone. This is illustrated in Figure 2 in the form of a streakline plot for set up no. $3(S=0.65)$. An example of the equivalence ratio gradients that are attained at the annular afterbody exit for each burner set up and at the same swirl and fuel injection level is displayed in Figure 3. Measurements of the fuel fraction at the afterbody annular jet exit were used to determine the equivalence ratio levels that emanate from the different premixing systems. The peak equivalence ratio varies from 1.85 to 1.3 and then to 0.95 for set ups no. 1, 2, and 3, respectively. The single disk with backward injection produces a much lower level of premixing in the incoming $\Phi$ profile and results in a burning mode with a much richer mixture similar to a nonpremixed configuration with yellowish flame colour. In contrast, as implied by the curves in this plot, both cavity systems appear more efficient in supplying a smoother stratified mixture profile close to the afterbody rim, where the leading flame front stabilization takes place.

The stability diagram of the three different burner arrangements is shown in Figure 4 in terms of the fuel blow off velocity $\left(U_{\mathrm{FBO}}\right)$ and the imposed swirl intensity for various central air supply velocities $\left(U_{C}\right)$. The near nonpremixed character of the single disk, with a rich fuel mixture supplied into the primary recirculation, an aspect that will be corroborated by the chemiluminescence images presented below, exhibits a somewhat lower blow off limit curve. However, this is attained at a significantly higher penalty regarding the emission of pollutants, and this is also discussed more extensively later in this section. On the other hand, the plots of Figure 4 suggest that the three-disk, double cavity set up no. 3 is more resistant to lean blow off compared to the single-cavity system no. 2; at least one cavity is required to reach a minimum amount of premixing and maintain a successful stratification. Both the single and the double-cavity configurations achieve a sufficiently premixed and stratified output mixture quality. The $\Phi$ distributions obtained by the double cavity however, sustain levels that are closer to stoichiometric values (Figure 4), particularly in the vicinity of maximum strain near the rim stabilization region. The stretch extinction rate therefore, there is locally strengthened [3], thus accomplishing a flame condition that is more resistant to blow-off at the leaner fuel settings than the single-cavity case.

The simulated flow topologies of the primary stabilizing recirculation zones that are produced from the three arrangements are displayed in Figures 5(a), 5(b), and 5(c). By comparison to the single-cavity system (Figure 5(a)), the double cavity shortens the primary vortex (Figure 5(c)) particularly at the higher swirls implying shorter flame lengths in accord with its improved premixed character. The effect of stronger swirl is illustrated in Figure 5(d). As swirl intensity increases in set up no. 3 from 0.65 to 1.00 , a central recirculation zone (CRZ) now appears downstream and interacts with the basic afterbody vortex, as displayed in the streakline plots. Similar patterns have been corroborated by detailed LDV measurements in reference [11]. This twin vortex system is present in all three set ups, but the interaction appears stronger in the three-disk system. The level of interaction between these two consecutive recirculations depends on the swirl intensity, the relative flow rates between the various streams, and the burner system geometry, that is, the swirl tube offset; appropriate regulation of these parameters is likely to produce a more efficient mixing of combustion products and control of exhaust pollutants.

The variations in flame front disposition for the three set ups at a swirl level of 0.65 as obtained from the $\mathrm{CH}^{*}$ chemiluminescence imaging studies are shown in Figure 6(a). The single disk displays a much longer toroidal flame front (4 to $5 D_{b}$ ) with a moderate reacting activity that penetrates into the axis and extends well above the flanking flame front. Nonetheless, this flame disposition indicates that the backward (upstream) injection, even in the case of the single cylinder, results in a much shorter flame by comparison to the forward injection directly into the wake formation region, as customarily seen in standard non-premixed jetlike arrangements (e.g., [2]). In contrast, both the two- and three-disk set ups, with successively increased premixing and smoother stratification at the burner inlet, drastically reduce the flame height (up to $1.5 D_{b}$ ) producing a wider flame spread. It is noteworthy that at a peak inlet equivalence ratio of about 0.9 , the double-cavity burner produces a somewhat shorter and wider conical flame disposition than similar 


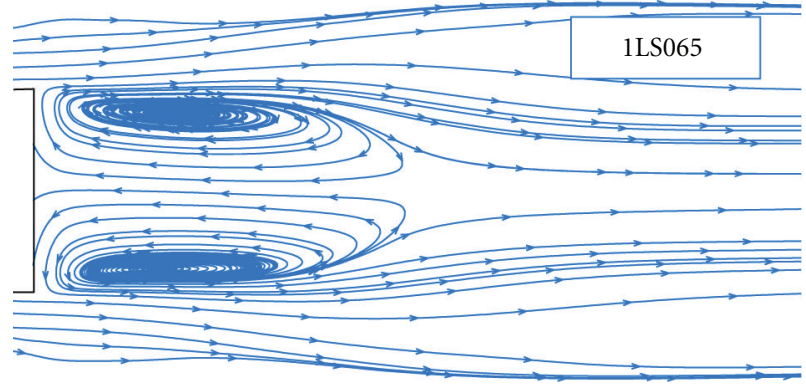

(a)

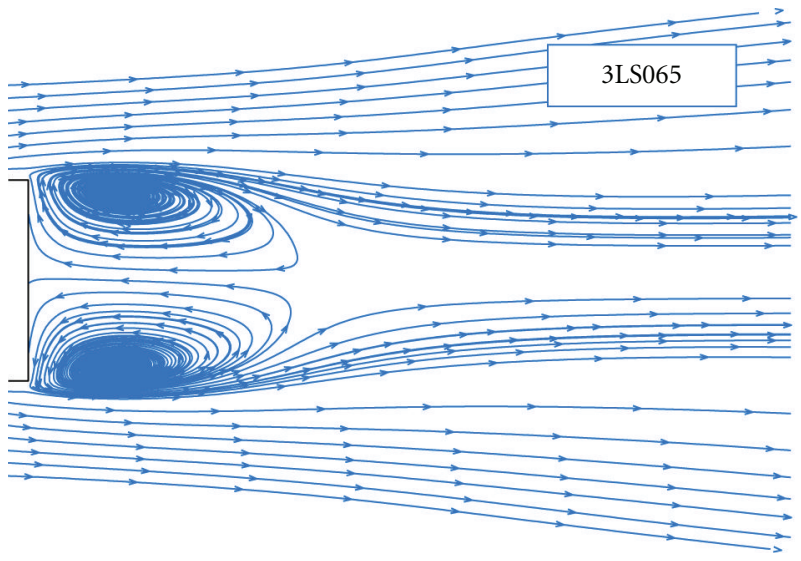

(c)

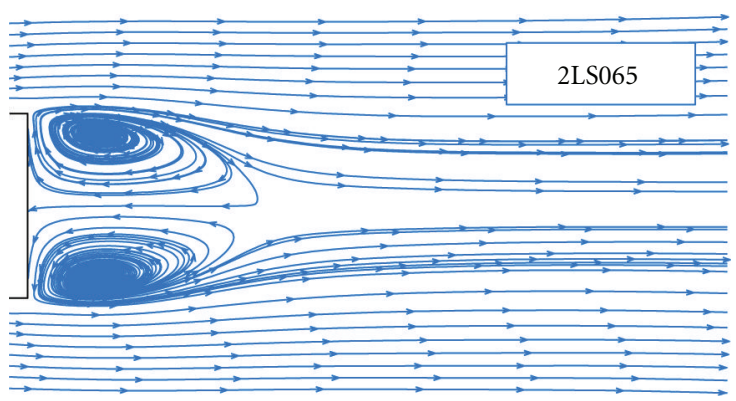

(b)

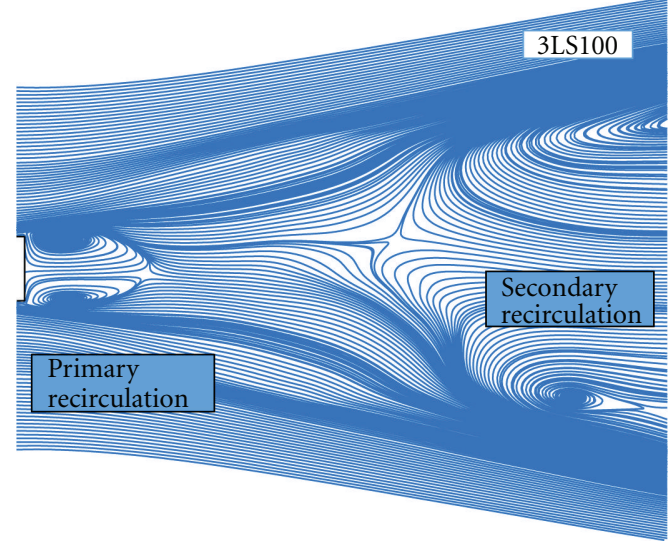

(d)

Figure 5: Axisymmetric wake flow fields for the three burner configurations.

fully premixed formulations (e.g., [5]). It should be noted that the single-disk system produced some longitudinal unsteadiness along the burner axis, while the single-cavity system exhibited a peripheral flame asymmetry and an attendant unsteadiness of the toroidal front. The addition of the forebody disk ( $\mathrm{C}$ in Figure 1(c)) in the double cavity system contributed to a stable and circumferentially uniform mixture development, a situation that proved conducive to better stability in the afterbody flame stabilization process.

The way the different set ups approach the lean blowoff limit is illustrated in Figures 6(b), 6(c), and 6(d) for the one-, two-, and three-disk set ups, respectively. The percentage from blow-off, defined by the parameter $\delta$ (see Table 1 ), is shown on these images. As fuel is reduced in the single disk, the toroidal flame initially shortens, and the on-axis reacting activity moderately intensifies and elongates before the final flame retraction at the lower fuel setting (Figure 6(b)). The unstable nature of the single cavity emerges more clearly as the fuel is gradually reduced and blow-off here appears in a more abrupt fashion (Figure 6(c)). The double cavity produces a circumferentially more symmetric and stable flame that approaches blow out by detaching a certain distance off the flame holder lip and also reverting from the conical to a cylindrical shape (Figure 6(d)).

Detailed measurements and simulations of radial temperature profiles for cases no. 1 and no. 3 and for two swirl intensities of 0 and 0.65 are presented in the comparisons of
Figures 7(a), 7(b), 7(c), and 7(d). Agreement is satisfactory with deviations found mainly in the shear layers emanating from the disk rims at the higher swirl intensity. The double cavity seemingly produces a faster wake development and spread of the temperature shear layers along the wake. Additionally, the impact of the swirl variation appears to be more significant in this case, and this allows for a better regulation of the primary zone conditions under operation with the double-cavity system. Overall, the variations exhibited in the profiles due to the introduction of the cavities and the increase in swirl intensity, are reproduced faithfully by the simulations in the mean temperature comparisons shown in Figures 7(a) and 7(b). The comparisons of the rms temperature profiles in Figures 7(c) and 7(d) suggest that the simulations captured all important trends and qualitative variations in these distributions with a somewhat poorer agreement achieved in the turbulent levels. The simulated results generally follow well the measured radial spread of the toroidal temperature shear layer close to the afterbody near wake region (Figures $7(\mathrm{c})$ and $7(\mathrm{~d}), x / D_{b}=0.06,1$ and 2). Deviations increase further along the axis (e.g., $x / D_{b}=3$ and 6 ) and for the higher swirl level as the interaction of the near wake development with the downstream emerging central recirculation zone (CRZ) gradually intensifies. It appears that the impact of this interference is stronger on the turbulence field as observed from the better accord in the mean fields (Figures 7(a) and 7(b)). 


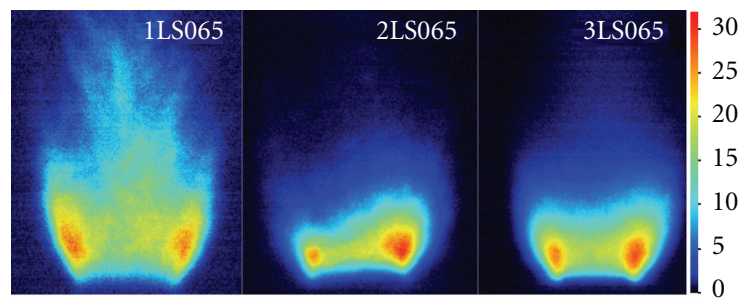

(a)

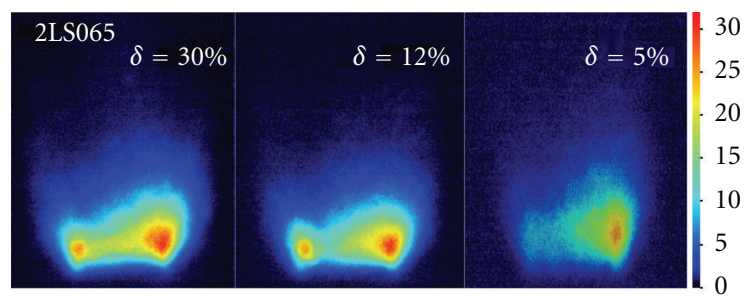

(c)

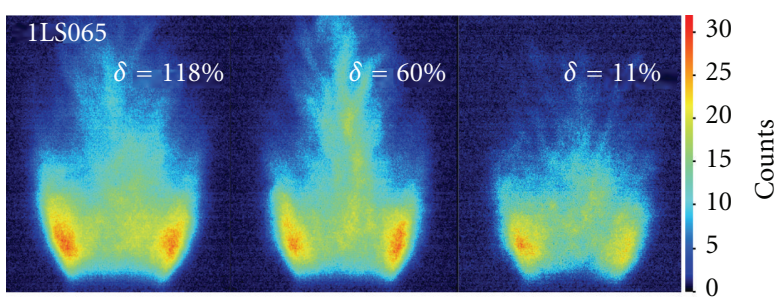

(b)

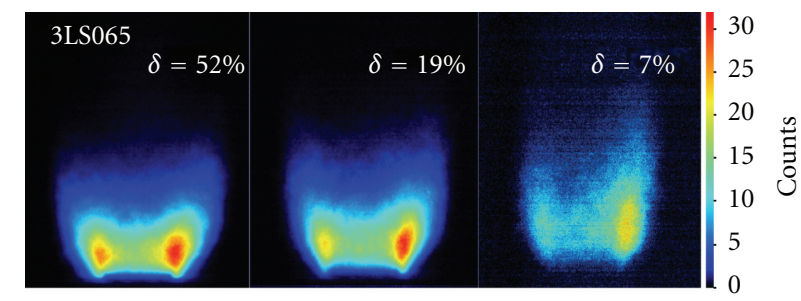

(d)

FIGURE 6: $\mathrm{OH}^{*}$ flame chemiluminescence images for the three burner set ups at various fuel injection levels (where $\delta$ denotes proximity to LBO, Table 1) and a swirl intensity of 0.65 .

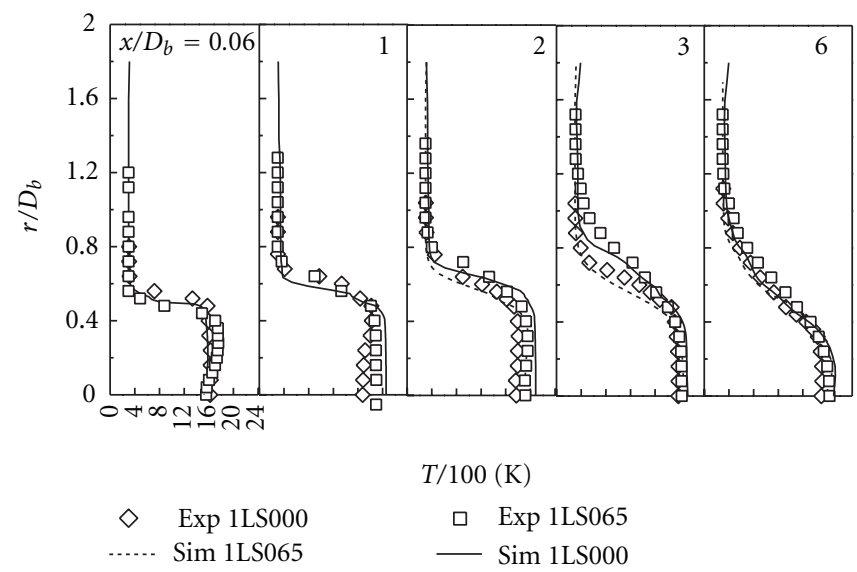

(a)

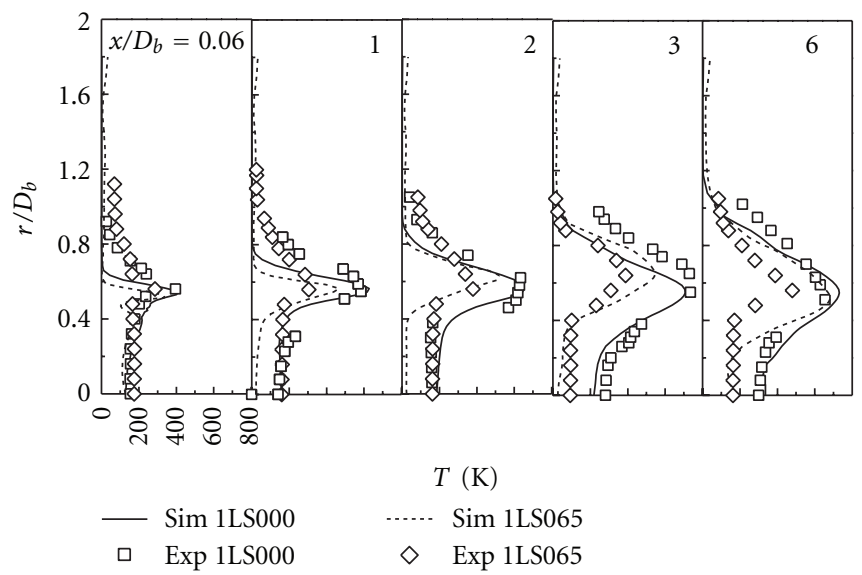

(c)

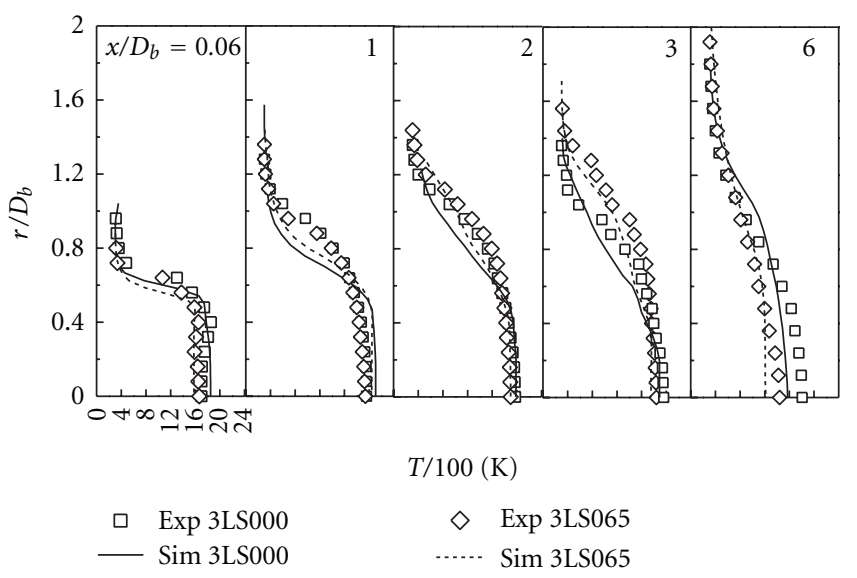

(b)

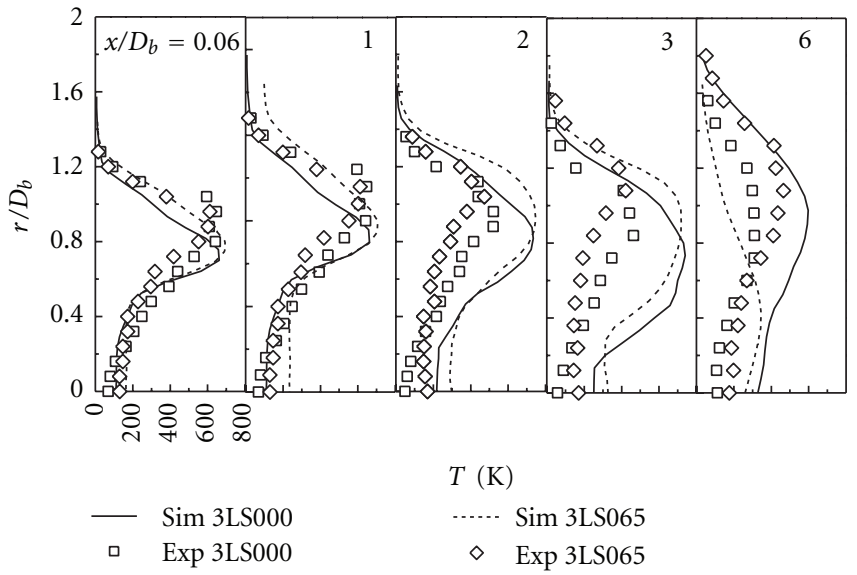

(d)

Figure 7: Comparisons between measurements and simulations of the time mean ( $a$ and $b$ ) and rms (c and d) radial temperature distributions throughout the wake for the single- and the three-disk burner set ups at swirl intensities of 0 and 0.65 . 


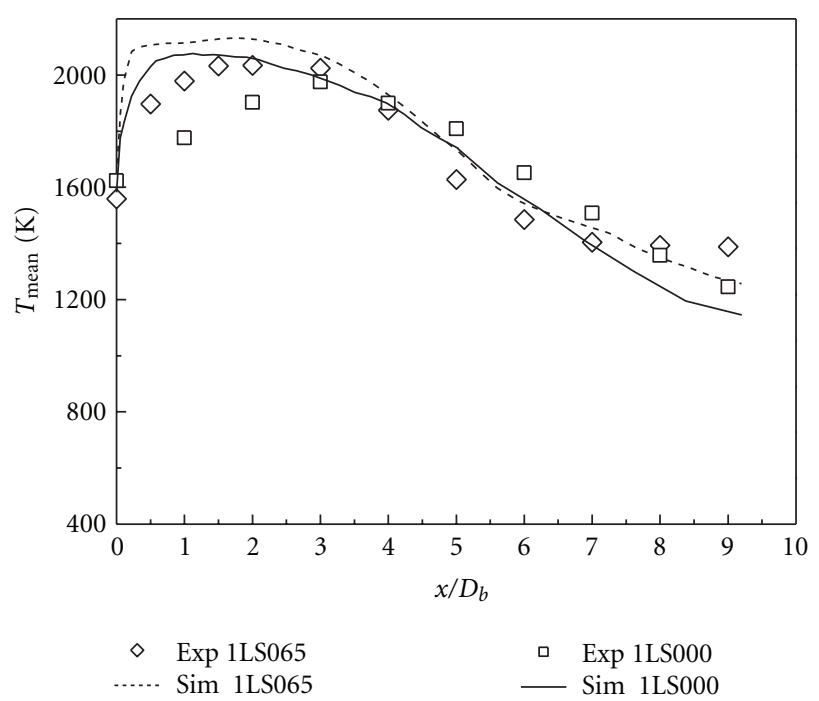

(a)

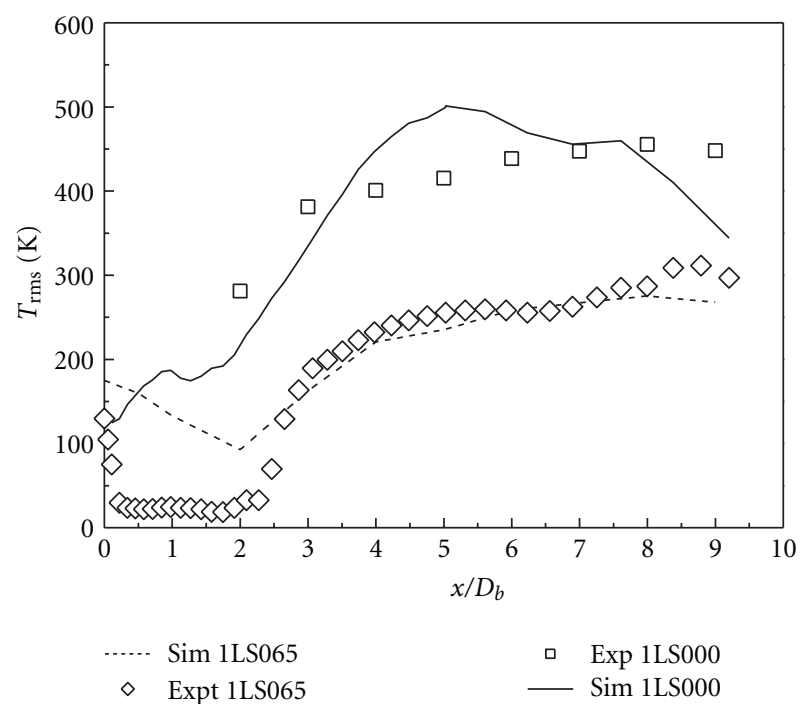

(c)

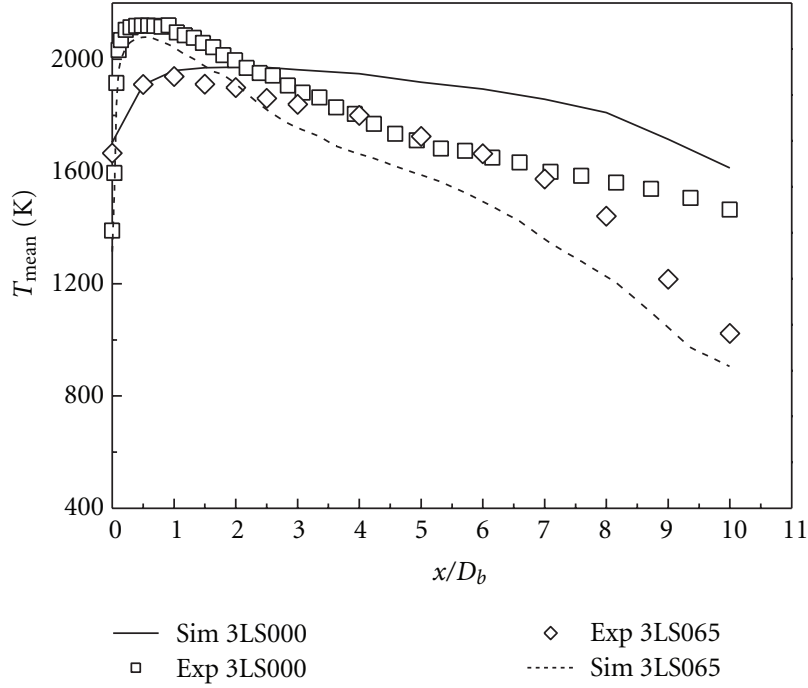

(b)

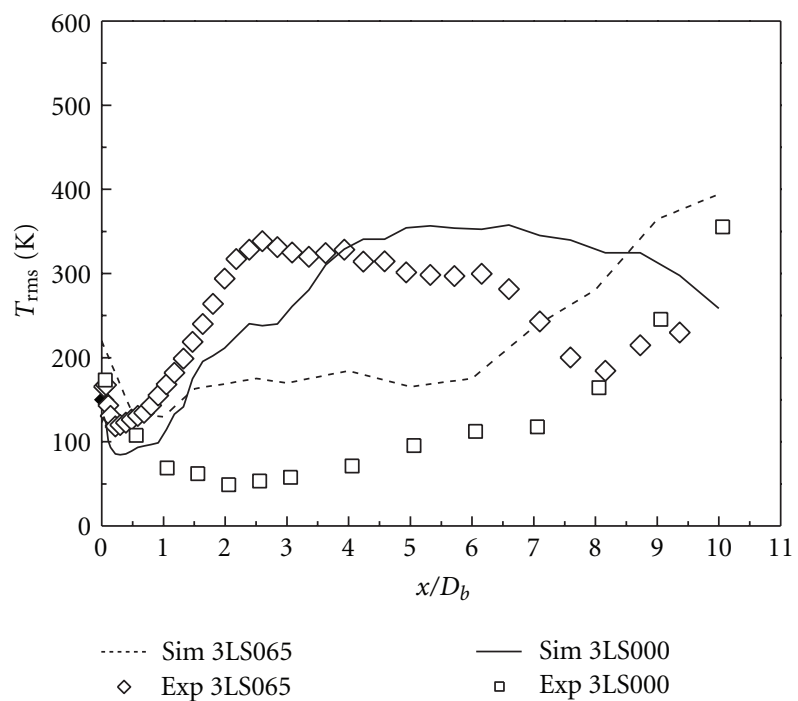

(d)

Figure 8: Center-line comparisons of the time mean ( $\mathrm{a}, \mathrm{b})$ and $\mathrm{rms}(\mathrm{c}, \mathrm{d})$ temperatures along the wake axis for the single- and the three-disk set ups and for swirl intensities of 0 and 0.65 .

The experimental and computational center-line development of the mean and fluctuating temperatures along the wake for the above respective cases is displayed in Figures $8(\mathrm{a}), 8(\mathrm{~b}), 8(\mathrm{c})$, and $8(\mathrm{~d})$. Apart from the region adjacent to the burner face, the mean temperature simulations agree both qualitatively and quantitatively in the near field, while differences increase, particularly for the double-cavity system, downstream of $x / D_{b}=4$ (Figures $8(\mathrm{a})$ and $8(\mathrm{~b})$ ) in the developing hot wake. The discrepancies seen within the single-disk system primary recirculation may also accrue from the global propane chemistry used that was mainly calibrated for partially premixed opposed jet flames and mixtures with $\Phi$ between 0.5 and 1.25, whereas these flames are operating with much higher inlet $\Phi$ 's (see Figure 3).
The double cavity also presents a faster temperature wake drop with respect to the single-disk set up, with this effect being more pronounced as the swirl increases, resulting in significantly shorter flame lengths.

Centre-line temperature peaks, which are closer to the centre-body accompanied by a faster cooling of the products along the wake, by comparison to the experiments, are seemingly produced by the model (Figures $8(\mathrm{a})$ and $8(\mathrm{~b})$ ). This could be attributed to the lack of a more complete heat transfer modelling near the wall (apart from the use of the simple radiation model discussed in Section 3.1), to the weaknesses in the EDC model (a slight improvement was achieved by the quasilaminar treatment although this was dependent on grid density), and to the performance 
of the global chemical scheme near the wall. The increased margin of error in the thermocouple measurements closer to the burner surface, as has been reported in [11], is yet another likely cause for these deviations. On the other hand, despite the encouraging checks of the quality index and the results of the mesh refinement tests (see Section 3.3), the influence of mesh resolution on these near wake results cannot be excluded with confidence. Admittedly, this was a very demanding geometry/flame configuration to tackle, since available computational resources had to be judiciously allocated both within the all important premixing cavities and in the two interacting recirculations (afterbody and swirl induced CRZ) along the developing wake. Nevertheless, the comparisons indicate that the present levels of mesh fineness clearly allow for a meaningful evaluation of the capability of the combined aerodynamic and combustion model to represent important trends and quantitative variations in the studied flame fields.

The flame and species topologies that were attained in the three arrangements are shown in Figures 9(a), 9(b), 9(c), and $9(\mathrm{~d})$. These illustrate the effective premixing of set up no. 3 resulting in a shorter flame with peak temperatures confined within a conical region in a fashion similar to premixed configurations. Production of $\mathrm{CO}$ and $\mathrm{NO}_{x}$ is also curtailed locally, in case no. 3, by 6 and 3.5 times, respectively, compared to the levels found in case no. 1 thus, emphasizing the benefits of the efficient and extensive partial premixing resulting in a smoother stratification extending over the lean region [13] (Figures 9(c) and 9(d)). These gains in emissions far outweigh the somewhat reduced fuel consumption of case no. 1 under lean operation, as might be implied by the lower blow-off limits of this arrangement in the stability diagrams of Figure 4 . Additionally, the radical $\mathrm{H}$ distributions (Figure 9(b)), signifying the location and extent of the reacting front in each case, indicate a more compact and opened up flame front for the three-disk set up that remains well-anchored at the afterbody rim and improves the stability and uniformity of the reacting zone (Figure 9(b)).

Comparisons between measured and computed global exhaust pollutants of $\mathrm{CO}$ and $\mathrm{NO}_{x}$ are shown in Figures 10 (a) and 9(b) for a range of swirl numbers and the same fuel settings. These represent a stringent test of the modelling methodology and encouragingly, the overall experimental trends have been followed satisfactorily. Carbon monoxide is consistently overestimated by the simulations across the swirl range, implying that an increased quenching effect is produced by the method. This could be attributed partly to a slower oxidation rate achieved by the turbulent chemistry employed and partly to an enhanced dilution effect resulting from the somewhat higher rates of entrainment produced by the model along the wake. The former scenario is more likely under operation with the single disk, where flames with inadequate premixing are obtained, while the latter is more plausible under the higher swirl intensities as a result of the CRZ activity. Discrepancies in the $\mathrm{NO}_{x}$ comparisons, shown in Figure 10(b), seem to be greater for the lower swirls in both burners. The model consistently underestimates the double-cavity burner $\mathrm{NO}_{x}$ values, while this behaviour is reversed for the single disk. The opposite trend seen in these two cases could be well associated with the variable model performance in the stratified short bluish doublecavity flames and the nonpremixed longer yellowish singledisk flames. A clearer interpretation of the $\mathrm{NO}_{x}$ results would possibly require more tests with other $\mathrm{NO}_{x}$ schemes as well to allow for a more definite conclusion to be reached for each case.

Nevertheless, it should be remarked that the indicated range of values obtained in the experiments and produced by the simulations is close to the levels that have been reported by Jachimowski and Wilson [21] for jet-stirred combustor studies with propane mixtures at similar conditions. Moreover it is evident that, in both cases, swirl has a beneficial effect on the overall emissions performance, although from previous discussions, it also seems to reduce moderately the stability envelope. The superiority of the double-cavity burner arrangement with the smoother and much leaner stratification that nonetheless sustains operational stability is clearly illustrated in these plots. Reductions of about three and four times in $\mathrm{CO}$ and $\mathrm{NO}_{x}$ emissions, respectively, are achieved by reverting to the two-cavity burner system operation (e.g., [3, 10, 13]). Additionally, this favourable behaviour seems to improve as swirl intensity is increased.

\section{Summary and Conclusions}

Experimental and computational investigations of turbulent reacting wakes established through staged fuel-air premixing in single- and double-cavity arrangements formed along one, two, or three concentric disks and stabilized in the downstream vortex region of the afterbody disk have been presented. The performance of the different burner assemblies with inlet fuel-air mixture stratification has been evaluated for lean and ultra-lean fuel settings under operation with a surrounding co-flow of swirling air.

Measurements and counterpart simulations illustrated the rich variety of flow and flame configurations that can be obtained under different swirls and burner arrangements. For all set ups, the impact of swirl on the reacting wake development appeared stronger at swirl intensities above 0.6, whereby a more evident reduction in peak temperatures and $\mathrm{NO}_{x}$ of up to 20 and $55 \%$, respectively, was obtained. All three configurations were operated with partial premixing of fuel with air and produced a more compact flame topology than traditional non-premixed set ups by up to $60 \%$, depending on set up, with appreciable gains in terms of emissions. The double-cavity, three-disk set up induced a significant stratification over a leaner range, while maintaining a flammable mixture across the flame front anchoring region at the disk rim. This proved more effective in stabilizing efficiently leaner and shorter flames which in turn produced lower peak temperatures and reduced $\mathrm{CO}$ and $\mathrm{NO}_{x}$ levels by three to four times with respect to the single-disk system.

The LES methodology with the adopted turbulent combustion and chemistry submodels proved capable of reproducing many significant features of these complicated partially premixed axisymmetric bluff-body flames. Discrepancies in the peak temperature and global species levels 


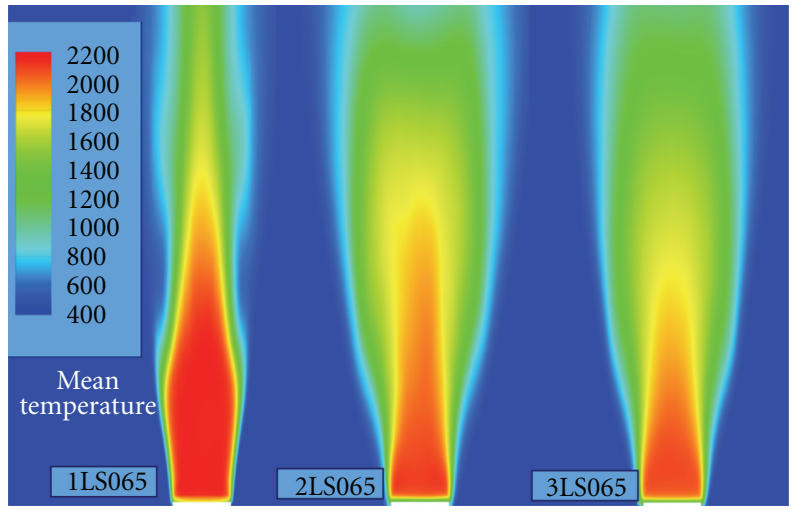

(a)

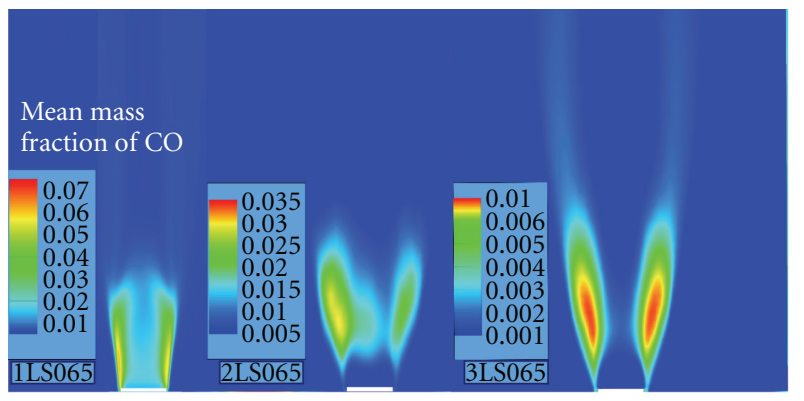

(c)

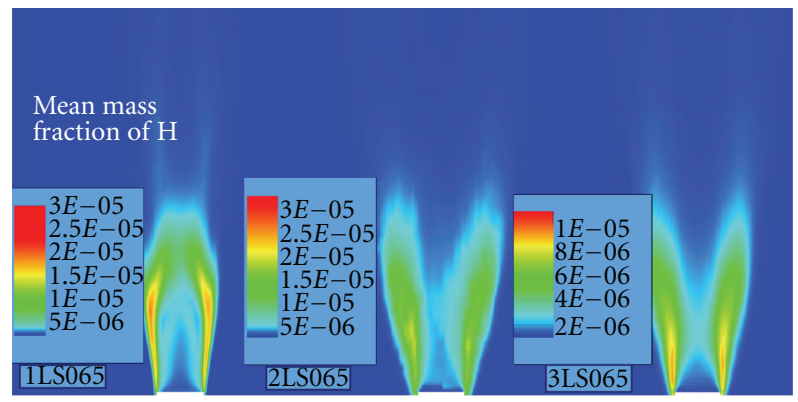

(b)

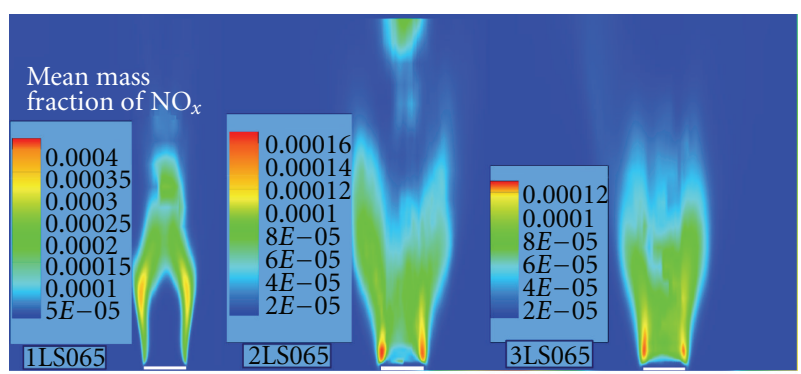

(d)

FIGURE 9: Simulated mean wake topologies for the three burner configurations, mean temperature (a) and mean mass fractions of radical $\mathrm{H}$ (b), $\mathrm{CO}(\mathrm{c})$ and $\mathrm{NO}_{x}(\mathrm{~d})$.

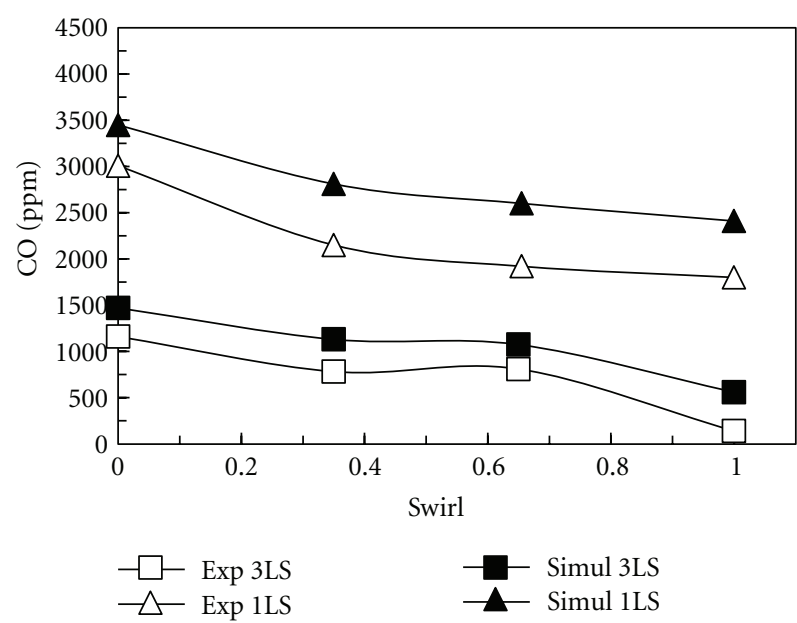

(a)

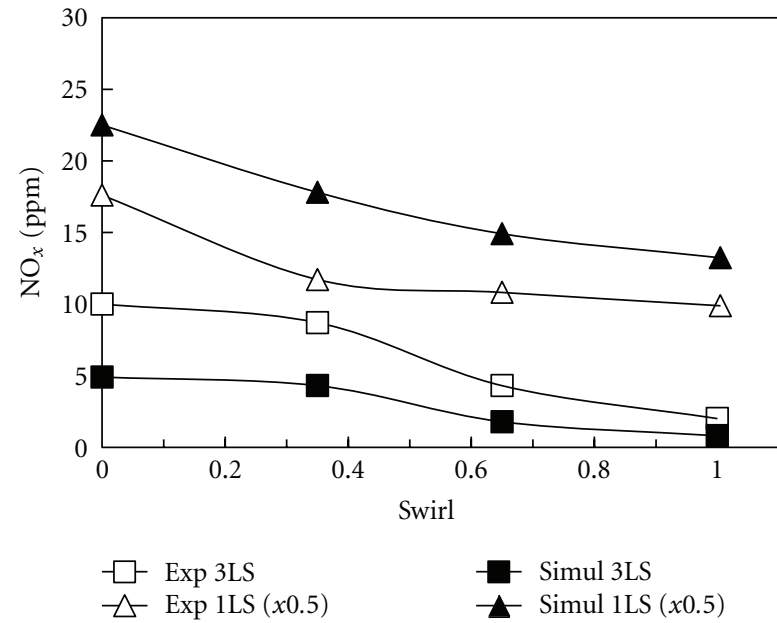

(b)

FIGURE 10: Experimental and computed major species global exhaust levels for the single- and the three-disk arrangements, (a) CO, and (b) $\mathrm{NO}_{x}$.

were of the order of $18 \%$, and $32 \%$ respectively, with maximum deviations found for the single-disk set up. The parametric simulations helped to expose the differences and similarities in the performance between the disk and cavity combinations that were examined. Additional tests and comparisons will allow for a more complete evaluation of the performance of the present or similar burner set ups and help contribute to their further development through an appropriate control and exploitation of the inlet mixture placement. 


\section{Acknowledgment}

This work was partly supported by the Research Council of the University of Patras.

\section{References}

[1] A. Cavaliere and M. De Joannon, "Mild combustion," Progress in Energy and Combustion Science, vol. 30, no. 4, pp. 329-366, 2004.

[2] A. R. Masri, P. A. M. Kalt, and R. S. Barlow, "The compositional structure of swirl-stabilised turbulent nonpremixed flames," Combustion and Flame, vol. 137, no. 1-2, pp. 1-37, 2004.

[3] D. Bradley, "Combustion and the design of future engine fuels," in Lean Combustion Technology and Control, D.-R. Derek, Ed., pp. 19-54, Academic Press, Elsevier, 2008.

[4] H. Wang, "Large eddy simulation/probability density function modeling of a turbulent $\mathrm{CH} 4 / \mathrm{H} 2 / \mathrm{N} 2$ jet flame," Proceedings of the Combustion Institute, vol. 33, pp. 1319-1330, 2011.

[5] S. Chaudhuri and B. M. Cetegen, "Blowoff characteristics of bluff-body stabilized conical premixed flames with upstream spatial mixture gradients and velocity oscillations," Combustion and Flame, vol. 153, no. 4, pp. 616-633, 2008.

[6] C. Duwig, K. J. Nogenmyr, C. K. Chan, and M. J. Dunn, "Large eddy simulations of a piloted lean premix jet flame using finite-rate chemistry," Combustion Theory and Modelling, vol. 15, no. 4, pp. 537-568, 2011.

[7] O. Lucca-Negro and T. O'Doherty, "Vortex breakdown: a review," Progress in Energy and Combustion Science, vol. 27, no. 4, pp. 431-481, 2001.

[8] M. Stohr, I. Boxx, C. Carter et al., "Dynamics of lean blowout of a swirl-stabilized flame in a gas turbine model combustor," Proceedings of the Combustion Institute, vol. 33, pp. 2953-2960, 2011.

[9] W. Lazik, T. Doerr, S. Bake, R. V. D. Bank, and L. Rackwitz, "Development of lean-burn low- $\mathrm{NO}_{x}$ combustion technology at rolls-royce deutschland," in Proceeding of ASME Turbo Expo, no. GT2008-51115, ASME, Berlin, Germany, 2008.

[10] K. T. Kim and S. Hochgreb, "Effects of non-uniform reactant stoichiometry on thermo-acoustic instabilities in a leanpremixed gas turbine combustor," Combustion Science and Technology, vol. 184, pp. 608-628, 2012.

[11] C. Xiouris and P. Koutmos, "An experimental investigation of the interaction of swirl flow with partially premixed disk stabilized propane flames," Experimental Thermal and Fluid Science, vol. 35, no. 6, pp. 1055-1066, 2011.

[12] M. S. Sweeney, S. Hochgreb, and R. S. Barlow, "The structure of premixed and stratified low turbulence flames," Combustion and Flame, vol. 158, no. 5, pp. 935-948, 2011.

[13] G. Kuenne, G. F. Seffrin, F. Fuest et al., "Experimental and numerical analysis of a lean premixed stratified burner using 1D Raman/Rayleigh scattering and large eddy simulations," Combustion and Flame, vol. 159, pp. 2669-2689, 2012.

[14] C. Bruno and M. Losurdo, "The trapped vortex combustor: an advanced combustion technology for aerospace and gas turbine applications," in Advanced Combustion and Aerothermal Technologies, N. Syred and A. Khalatov, Eds., pp. 365-384, Springer, 2007.

[15] C. Xiouris and P. Koutmos, "Fluid dynamics modeling of a stratified disk burner in swirl co-flow," Applied Thermal Engineering, vol. 35, pp. 60-70, 2012.
[16] I. S. Ertesvåg and B. F. Magnussen, "The Eddy dissipation turbulence energy cascade model," Combustion Science and Technology, vol. 159, no. 1-6, pp. 213-235, 2000.

[17] G. Paterakis and P. Koutmos, "An investigation of the characteristics of planar and axisymmetric bluff body stabilized turbulent stratified flames close to lean blow off," in Proceedings of the Engineering Turbulence Modelling and Experiements 9, ETMM9, Salonika, Greece, June 2012.

[18] P. Koutmos and K. Souflas, "A study of slender bluff body reacting wakes formed by con-current or counter-current fuel injection," Combustion Science and Technology, vol. 184, pp. 1343-1365, 2012.

[19] D. Trimis, Verbrennungsvorgange in Porosen Inerten Medien, ESYTEC, Elangen, Germany, 1996.

[20] S. B. Pope, "Ten questions concerning the large-eddy simulation of turbulent flows," New Journal of Physics, vol. 6, pp. 35-47, 2004.

[21] C. J. Jachimowski and C. H. Wilson, "Chemical kinetic models for combustion of hydrocarbons and formation of nitric oxide," Tech. Rep. NASA TP-1794, 1980. 

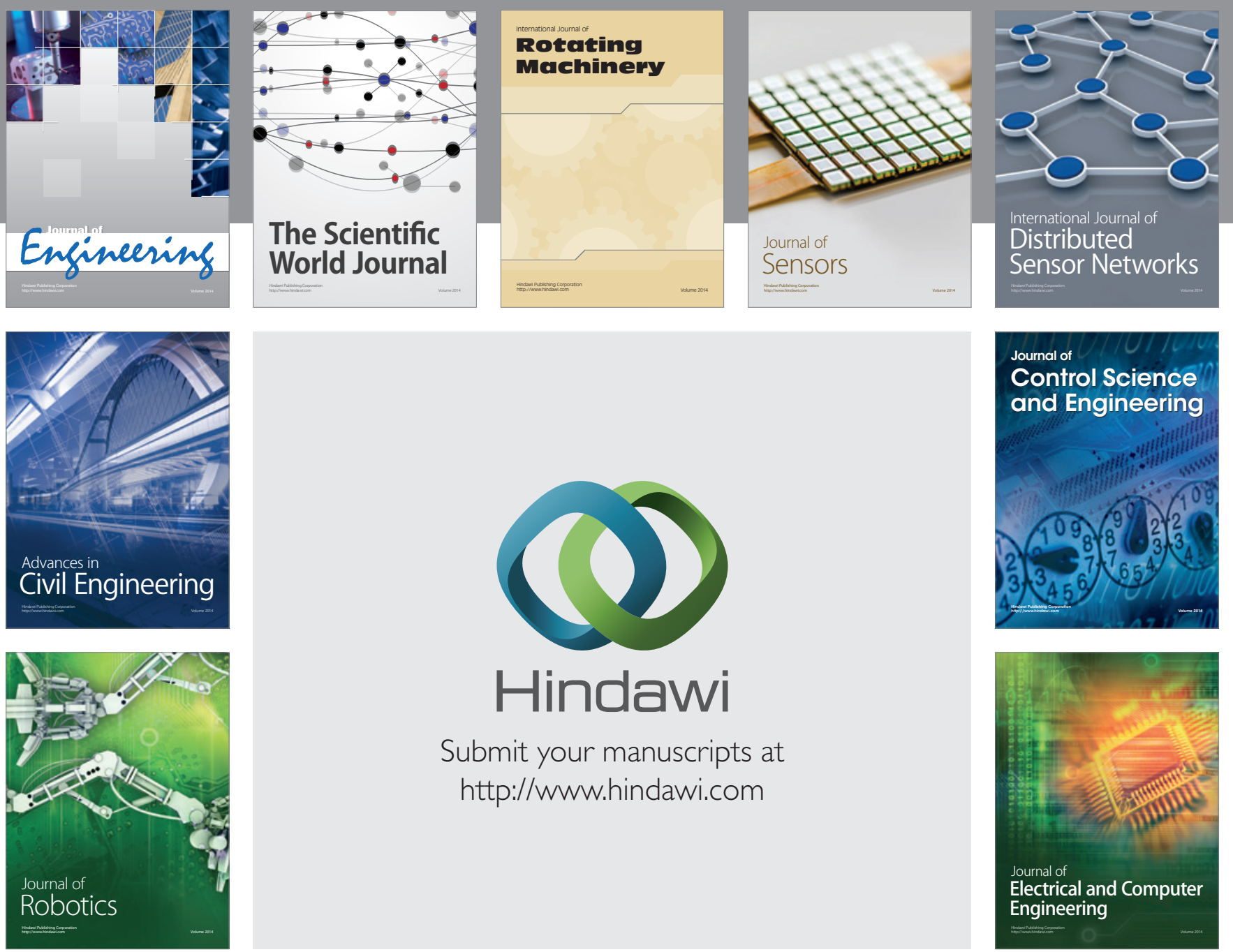

Submit your manuscripts at

http://www.hindawi.com
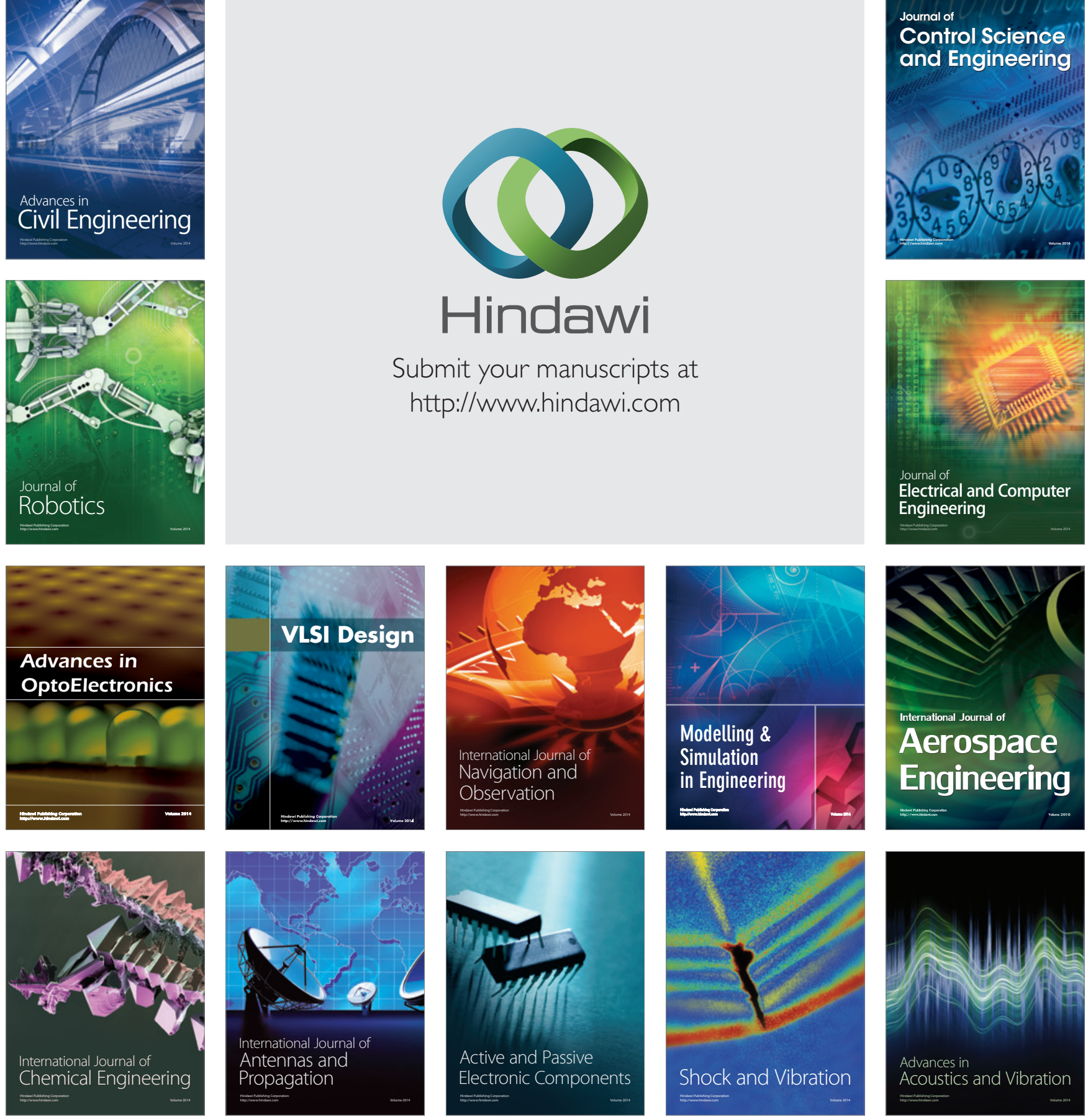